\title{
Thief zone identification based on transient pressure analysis: a field case study
}

\author{
Daolun $\mathrm{Li}^{1,3} \cdot$ Jinghai Yang ${ }^{2} \cdot$ Detang $\mathrm{Lu}^{3}$
}

Received: 9 January 2014/Accepted: 22 March 2015/Published online: 17 April 2015

(c) The Author(s) 2015. This article is published with open access at Springerlink.com

\begin{abstract}
Thief zones are generally a channel with high absolute permeability. However, a not very strict theory analysis shows that neglecting the effect of variant of water saturation on formation of thief zone in mature reservoirs cannot explain some thief zones in mature reservoirs. This paper focuses on the effect of water saturation on thief zone development for wells in mature reservoirs with areal permeability heterogeneity. The water saturation increases in high permeable channel with water passing through, which gradually leads to higher value of water phase relative permeability, and thus leads to higher value of effective permeability $k \cdot k_{\mathrm{rw}}$ in the high permeable channel than that in surrounding reservoir. The gradually increasing difference of effective permeability $k \cdot k_{\mathrm{rw}}$ between permeable channel and surrounding reservoir would be the main reason for formation of thief zones in mature reservoir. The oil-water two-phase numerical model based on PEBI (perpendicular bisection) gridding is used to study effect of variant of water phase saturation on formation of thief zone. We find that relatively small pressure change and not obvious bump in pressure derivative curves during shut-in are indicators of thief zones in mature reservoirs. This finding provides a further understanding for the thief zone development and also gives us a guidance to interpret the
\end{abstract}

Daolun Li

ldaol@ustc.edu.cn

1 Hefei University of Technology, Hefei, China

2 Logging \& Testing Services Company, Daqing Oilfield Co. Ltd, Daqing, China

3 University of Science and Technology of China, Hefei, China buildup pressure data in the mature sandstone reservoirs. A field case study verifies the effectiveness of the new explanation.

Keywords Thief zone - Uneven saturation distribution · Transient pressure $\cdot$ Relative permeability $\cdot$ Well test

\section{Introduction}

As the major oil and gas fields are becoming more and more mature, wells start to experience water breakthrough problems due to the presence of the thief zone. As early in 1975 (Felsenthal and Gangle), the thin layers penetrated by water were called "thief zones" because they draw off large quantities of water and thereby impair the economics of the waterflood operation. In that paper, a thief zone is defined as a relatively thin layer comprising $5 \%$ or less of the net pay thickness and taking more than $25 \%$ of the injected water in a given well. The thief zone, also called "super permeability" zone or "speed zone" in some other studies, is defined by Bane as laterally continuous stratigraphic unit of relatively high permeability and large pore radius, which has approached residual oil saturation (Bane et al. 1994).

Many factors can lead to the formation of the thief zone according to the current studies (Felsenthal and Gangle 1975; Li et al. 2008; Azim et al. 2003; Shawket et al. 2010; Feng et al. 2010). Firstly, the widely accepted view is the serious reservoir heterogeneity. Areal and vertical reservoir heterogeneity is considered as main causes of early water breakthrough (Shawket et al. 2010). Viewed as two-layer infinite homogenous reservoir, Feng et al. (2010) consider the thief zone as a high permeability layer with residual oil saturation and provides an approach to thief zone identification based on the combination of wellbore pressure and

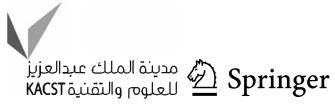


layer flow rates. Layer thickness and gravity are also considered as factors of formation of thief zones. Secondly, in the fractured reservoirs, the fracture connecting the producer and injector will lead to water breakthrough and easily forms the thief zone ( $\mathrm{Li}$ et al. 2008; Azim et al. 2003). Furthermore, some papers mention that the porosity structure of reservoirs will change distinctly after longterm water flooding due to the dissolution and erosion of the formation, which forms the high porosity and high absolute permeability channel and contributes to formation of thief zones ( $\mathrm{Li}$ et al. 2008; Feng et al. 2010).

There have been many dynamic monitoring methods used to identify thief zones. Chetri et al. (2003) and Azim et al. (2003) used core, logs and injection profiles/production profiles to understand the vertical distribution of the water injected/oil produced and to identify thief zones. Feng et al. (2010) used well test method to quantitatively identify thief zones between wells, and to estimate parameters of the reservoir. Li et al. (2008) presented a methodology of recognizing different types of thief zones in the Mauddud carbonate reservoir through integration of high-resolution image logs with PLT, NMR and conventional logs, and detected the thief zones in carbonate reservoirs by integrating borehole image with dynamic measurements.

Although many studies have focused on the thief zone formation caused by areal or vertical absolute permeability heterogeneity, the effect of increasing water saturation induced by the areal permeability heterogeneity on the formation of thief zones is not fully studied. Bane et al. (1994) only refer to approached residual oil saturation of thief zone and Feng et al.(2010) just take approached residual oil saturation as assumption. Furthermore, Zhong et al. (2007) find that some wells with the thief zone characteristics do not have water breakthrough at early stage; however, when injection pressure decreases at the late stage, injection rate of these wells is higher than before, which obviously cannot be explained by the absolute permeability difference.

Therefore, this paper focuses on the formation of thief zone in mature reservoirs caused by variant of water phase saturation induced by the areal permeability heterogeneity during water flood. At first, a not very strict theory analysis shows that negligence of the uneven water saturation distribution cannot explain the thief zone appearing in mature reservoirs after many years development. Then, numerical simulation based on PEBI grids studies the characteristic of the pressure transient response for the wells located in the thief zone with high water saturation. Finally, a case study in Daqing oilfield is studied.

\section{Absolute permeability difference is not the only contribution to formation of thief zone}

The absolute permeability is a property of the porous medium and a measure of the capacity of the medium to transmit fluids, and thus is always considered as main contribution to formation of thief zones. Some papers report the absolute permeability contrast between thief zones and surrounding ordinary reservoirs. For example, the permeability in thief zone is more than 20 times (Feng et al. 2010) or 30-500 times (Luo et al. 1999) of that in the ordinary reservoir.

Twenty times of permeability difference means the throat of porous medium in the thief zone is much bigger than that in the ordinary reservoir. In pore network model of porous media, the flow channel is composed of many cells of pore bodies connected by throats. If the pore is considered as a capillary, according to the Hagen-Poiseuille formula, we have

$Q=\left(p_{i}-p_{j}\right) \frac{\pi r^{4}}{8 \mu L}$.

The flux in capillary is proportional to radius' fourth power, and permeability is proportional to radius' second power. Therefore, if the permeability in the thief zone is more than 20 times of that in the ordinary reservoir, the radius of the porous throat in thief zone will be 4 times bigger than the porous throat radius in ordinary reservoirs. If the size of porous throats increases 4 times, the size of pore bodies will increase too. Therefore, not very strictly, the porosity of the thief zone is about more than 10 times bigger than the porosity of the normal reservoir, which may be not true.

Through the simple and not very strict analysis, we know that only absolute permeability difference cannot explain formation of some thief zones, especially for the thief zone formed at late development in mature reservoirs, and there should be other mechanisms for the formation of this kind of thief zones.

\section{The formation of the thief zone caused by uneven water saturation distribution}

\section{Permeability heterogeneity induces water saturation heterogeneity during water flooding}

Because of the existence of the high permeable channel and oil and water viscosity difference, when water is injected for reservoir pressure maintenance and increasing oil recovery factor, more water will flow through the high permeable zone, and water saturation in the high permeable zone will increase more quickly than water saturation in other places. After decades of water flooding, water saturation difference between the high permeable zone and the surrounding reservoir becomes bigger and bigger. Therefore, permeability heterogeneity induces water saturation heterogeneity during water flooding.

Figure 1 shows the permeability distribution of a channel sandstone reservoir with two wells. The permeability of 


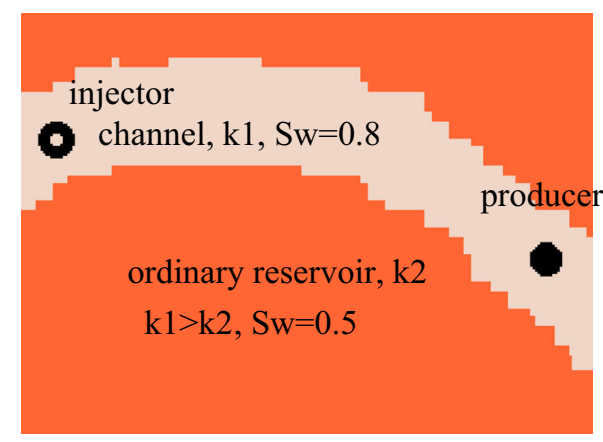

Fig. 1 Permeability heterogeneity and saturation distribution heterogeneity

the channel and the ordinary reservoir is noted as $k_{1}$ and $k_{2}$, respectively, and $k_{1}$ is larger than $k_{2}$. Due to permeability difference and viscosity difference, when water is injected to displace oil, water phase goes faster in the channel, and thereby water saturation in the channel increases faster than in other places. Water saturation difference between the channel and its adjacent region becomes larger and larger with time. Therefore, permeability heterogeneity strengthens the uneven distribution of water saturation.

\section{Relative permeability difference is an important contribution to some thief zones}

Effective permeability of any reservoir fluid is a function of the reservoir fluid saturation and the wetting characteristics of the formation. $k_{\mathrm{o}}$ and $k_{\mathrm{w}}$ are the symbols for the effective permeability to oil and water, respectively.

When two fluids flow at the same time, the relative permeability of each phase at a specific saturation is the ratio of the effective permeability of the phase to the absolute permeability.

$k_{\mathrm{ro}}=\frac{k_{\mathrm{o}}}{k}$

$k_{\mathrm{rw}}=\frac{k_{\mathrm{w}}}{k}$

The Darcy's law for oil-water two-phase system can be written as:

$u_{\mathrm{o}}=-\frac{k \cdot k_{\mathrm{ro}}}{\mu_{\mathrm{o}}}\left(\nabla p_{\mathrm{o}}+\rho_{\mathrm{o}} g\right)$

$u_{\mathrm{w}}=-\frac{k \cdot k_{\mathrm{rw}}}{\mu_{\mathrm{w}}}\left(\nabla p_{\mathrm{w}}+\rho_{\mathrm{w}} g\right)$

The mobility for oil phase and water phase is mainly determined by $\frac{k \cdot k_{\mathrm{ro}}}{\mu_{\mathrm{o}}}$ and $\frac{k \cdot k_{\mathrm{w}}}{\mu_{\mathrm{w}}}$, respectively. For water phase, effective permeability $k \cdot k_{\mathrm{rw}}$ decides the mobility at a specific place of a reservoir. In the mature reservoir, when the water saturation is big, water phase relative permeability increases dramatically, especially when the

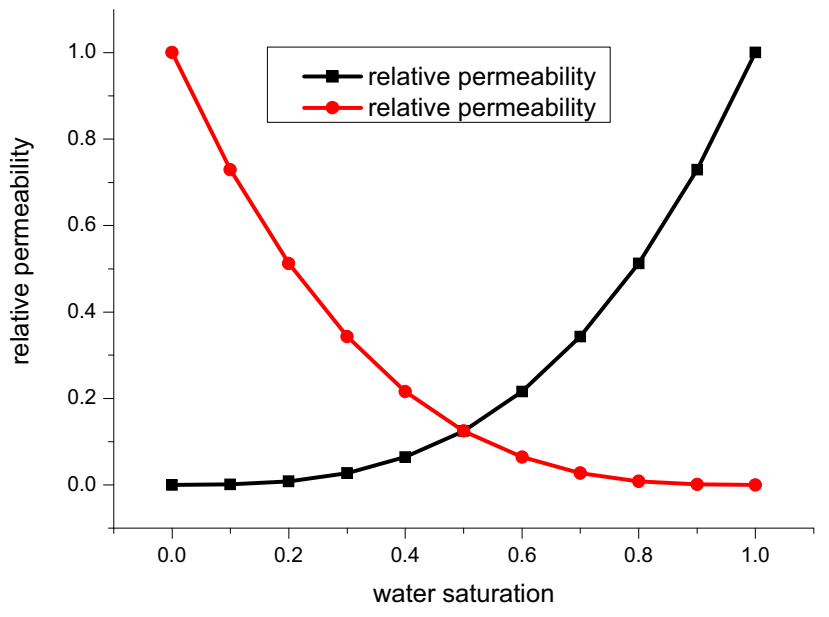

Fig. 2 A typical relative permeability curves

water saturation is approaching to the maximum water saturation.

Figure 2 shows the relative permeability curves, which are calculated from correlation. Suppose that absolute permeability difference between the channel and the other place is twice, and after long time water flooding, water saturation $S_{\mathrm{w}}$ in the channel is 0.8 and 0.5 in the other place (Fig. 1). According to Fig. 2, the water saturation difference leads to that water relative permeability in the channel is about 4 times of that in the ordinary reservoir. Combining absolute permeability difference with the relative permeability difference, the effective permeability in the channel is about 8 times of that in the ordinary reservoir.

Therefore, the magnitude of difference of water phase relative permeability between the thief zone and the ordinary reservoir is more likely the main contribution to the formation of some thief zones in mature reservoirs, while absolute permeability heterogeneity is a relatively minor factor, but it is the initial factor.

\section{Effect of water saturation on transient pressure response based on numerical simulations}

UST numerical well test software is based on PEBI gridding ( $\mathrm{Li}$ et al. 2014; Zha et al. 2008; Heinemann et al. 1991; Mahani and Evazi 2010). The software is used to generate the numerical simulation to study effect of water phase saturation on the formation of thief zone in the mature reservoir.

The reservoir size is $890 \times 710 \mathrm{~m}$ with 5 wells as shown in Fig. 3. The initial oil saturation is 0.55 and Fig. 2 gives the relative permeability curves.

Table 1 shows the reservoir and fluid parameters. The oil and water compressibility are the same in order to easily keep balance between injection and production 


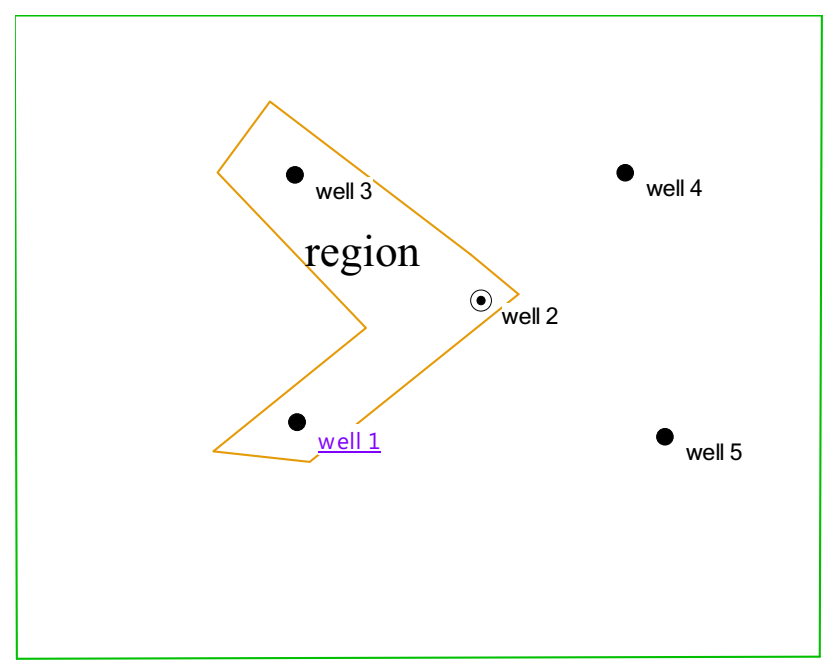

Fig. 3 Reservoir and wells

Table 1 Simulation parameters

\begin{tabular}{ll}
\hline Name & Value \\
\hline Initial pressure & $2900(\mathrm{psi})$ \\
Initial oil saturation & 0.55 \\
Reservoir thickness & $10(\mathrm{~m})$ \\
Horizontal permeability & $500(\mathrm{mD})$ \\
Porosity & 0.2 \\
Oil and water compressibility & $1.378 \mathrm{e}-006(1 / \mathrm{psi})$ \\
Rock compressibility & $1.034 \mathrm{e}-006(1 / \mathrm{psi})$ \\
Viscosity of oil & $8.6(\mathrm{mPa} \mathrm{s})$ \\
Viscosity of water & $0.6(\mathrm{mPa} \mathrm{s})$ \\
\hline
\end{tabular}

Table 2 Parameters of the region

Case $1 S_{\mathrm{o}}=0.1, k=1000 \mathrm{mD}$ in the region Ratio of $k \cdot k_{\mathrm{rw}}$ is 15.4

Case $2 S_{\mathrm{o}}=0.3, k=1000 \mathrm{mD}$ in the region Ratio of $k \cdot k_{\mathrm{rw}}$ is 7.26

Case 3 Even distribution of $S_{\mathrm{o}}$ and $k=1000 \mathrm{mD}$ in the region

Ratio of $k \cdot k_{\mathrm{rw}}$ is 2

Case 4 No region

Ratio of $k \cdot k_{\mathrm{rw}}$ is 1

In order to simulate the thief zone, we suppose that a region with different saturation and permeability exists in the reservoir as shown in Fig. 3. Four cases are considered to study the characteristics of transient pressure response as shown in Table 2. The ratio of $k \cdot k_{\mathrm{rw}}$ in the region to $k \cdot k_{\mathrm{rw}}$ in the other place is also displayed in Table 2 . The ratio of $k \cdot k_{\mathrm{rw}}$ is biggest in case 1 . The case 3 means that only permeability difference exists between the region and other place. The case 4 means even permeability distribution.

The numerical simulation can generate the series $\left\{\left(t_{j},-\right.\right.$ $\left.\left.p_{w f, j}\right) j=0,1, \ldots, m\right\}$, where $\left(t_{0}, p_{\mathrm{wf}, 0}\right)$ means initial time and initial reservoir pressure. Definition of the pressure change and pressure derivative is as follows: $d p=\left|p_{w f, j}-p_{w f, 0}\right|, 1, \ldots, m$

$\mathrm{d} p^{\prime}=\left|\frac{\mathrm{d} p_{w f}}{\mathrm{~d} t}\right| t$

Then, we draw the curves of pressure change and pressure derivative to study the characteristics of pressure change and pressure derivative for the well located in the thief zone.

Effect of water saturation on transient pressure response under constant pressure boundary condition

The well 1 produces for 60 days at rate of $100 \mathrm{~m}^{3} /$ day and then shuts in for 3 days to see the buildup pressure response. The well 2 is injection well and the others are production wells. The details are given in Table 3 .

The pressure change and pressure derivative of the well 1 during pressure buildup period is shown in Fig. 4.

Figure 4a shows that bumps in case 3 and case 4 are more obvious than that in case 1 and case 2, which indicates that high ratio of $k \cdot k_{\mathrm{rw}}$ between the region and other place (15.4 for case 1, 7.26 for case 2 ) can slow down the bump of the pressure derivative at the early stage.

Another characteristic of transient pressure response is that high ratio of $k \cdot k_{\mathrm{rw}}$ between the region and other place can lead to low pressure change as seen Fig. 4a. The pressure change of well 1 is 49, 68, 146 and 250 psi for case 1 , case 2, case 3 and case 4 , respectively. The fitted function for the pressure change during pressure buildup period shown in Fig. $4 \mathrm{~b}$ is $y=148.78403 \times \exp (-x / 2.59781)+148.78403 \times \exp (-$ $x / 2.59781)+48.20739$, which means that pressure change is an exponential function of the ratio of the effective permeability.

Therefore, the characteristic of transient pressure for the well in thief zone is the small pressure change and relatively flat derivative at early stage.

Effect of water saturation on transient pressure response under closed boundary condition

When the boundary condition is closed and other data are as the same as in "Effect of water saturation on transient pressure response under constant pressure boundary condition", the pressure change and pressure derivative of the well 1 during pressure buildup period are shown in Fig. 5

Figure 5a shows that although the boundary condition changes, the curves of case 1 and 2 almost have the same characteristic as in Fig. 4a before the boundary effect appears. Figure $5 \mathrm{~b}$ shows that the pressure change during pressure buildup period is an exponential function of the ratio of the effective permeability. 
Therefore, Fig. 5 again means that large difference of $k \cdot k_{\mathrm{rw}}$ between the region and ordinary reservoir leads to small pressure change and low bump of the pressure derivative, which would be an indicator of thief zone.

Transient pressure response with wells operated at constant rate production

All the wells in this section are under constant rate production, and the magnitude is shown in Table 4.

Figure $6 \mathrm{a}, \mathrm{b}$ gives the transient pressure response for the well 1 with constant pressure boundary condition and with closed boundary condition, respectively, which also shows that large difference of $k \cdot k_{\mathrm{rw}}$ between the region and ordinary reservoir leads to small pressure change and relatively low bump in the curves of pressure derivative.

Figures 4, 5 and 6 show that when ratio of $k \cdot k_{\mathrm{rw}}$ in the region to $k \cdot k_{\mathrm{rw}}$ in ordinary reservoir is 15.4 for case 1 and 7.26 for case 2, characteristics of thief zones are obvious. Because the ratio of $k$ in the region to $k$ in ordinary reservoir is only two, the effective permeability difference is mainly caused by water saturation, which is induced by permeability heterogeneity. Therefore, the numerical

Table 3 Well data

\begin{tabular}{lll}
\hline $\begin{array}{l}\text { Well } \\
\text { name }\end{array}$ & $\begin{array}{l}\text { Well Details } \\
\text { type }\end{array}$
\end{tabular}

Well 1 Producer Produces at constant rate of $100 \mathrm{~m}^{3} /$ day for 60 days, and then shuts in for 3 days

Well 2 Injector Injects at constant BHP of 2973 psi for 63 days

Other Producer Produces at constant BHP of 1015 psi for Wells 63 days

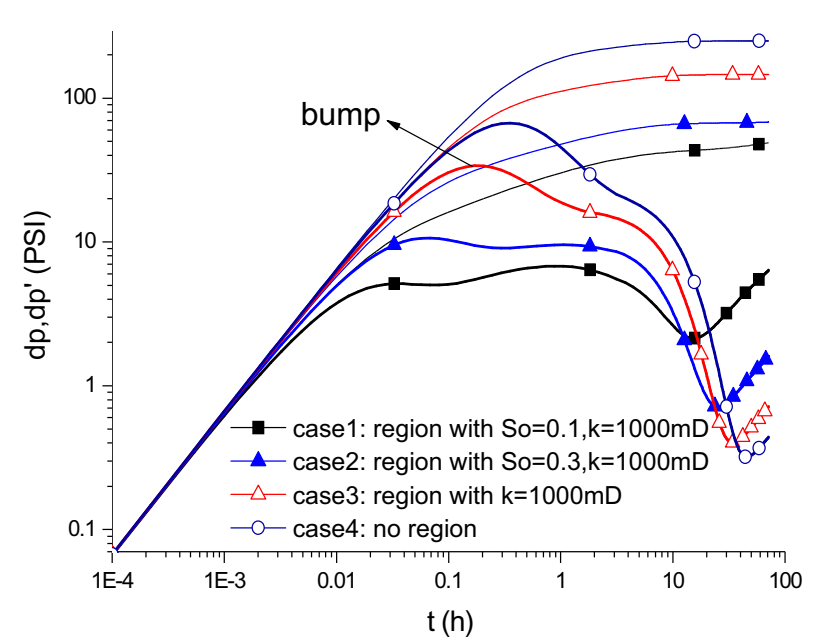

(a) simulation shows that the uneven water saturation could be main contribution to the formation of thief zones in mature reservoirs.

\section{Case study}

\section{Field description}

The Daqing Oilfield is a huge complex that consists of many individual oil fields and is located in the Songliao basin in northeastern China. It is a fluvial deposits formed under continental sedimentary environments during the Lower Cretaceous period. Relatively good development result has been achieved since its development. South block 5 is located in Sartu, one of oil fields with reservoir temperatures of -36.2 to $37.4{ }^{\circ} \mathrm{C}$. Crude oil in the Sartu formations is slightly heavy with a viscosity ranging from 5.0 to $20.0 \mathrm{mPa}$ s under reservoir conditions, depending on the crude oil composition, solution gas content and reservoir temperatures. The porosities range from 18 to $28 \%$.

South block 5 was brought into development in 1965 as major reservoirs in Sartu. Since then two times of infilling adjustment had been conducted. In 1984 of first infilling, $250 \times 300 \mathrm{~m}$ inverted nine spots area injection pattern is adopted. In 1996, secondary infilling adjustment adopts irregular five spots area water injection pattern with well spacing of $250 \mathrm{~m}$.

For a majority of sand stone oil reservoir, large amounts of oil still remains unrecovered even after extensive water flood, due to reservoir heterogeneity and viscosity difference between oil and water. The reservoir heterogeneity could lead to water channels formed in high permeable zones, and result in that injected water simply bypasses the

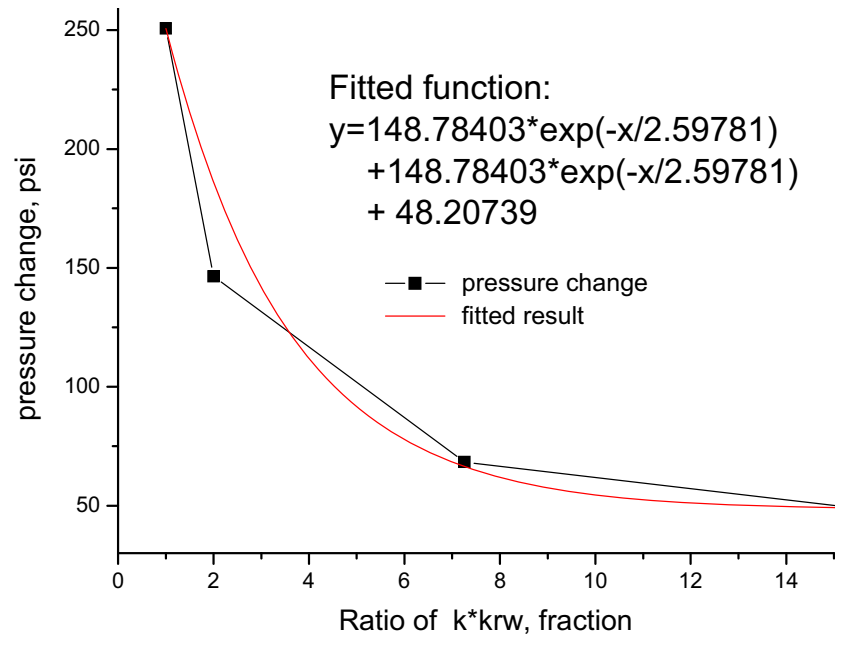

(b)

Fig. 4 Transient pressure response and pressure change. a Transient pressure response, $\mathbf{b}$ pressure change vs ratio of $k \bullet k_{\mathrm{rw}}$ during buildup 


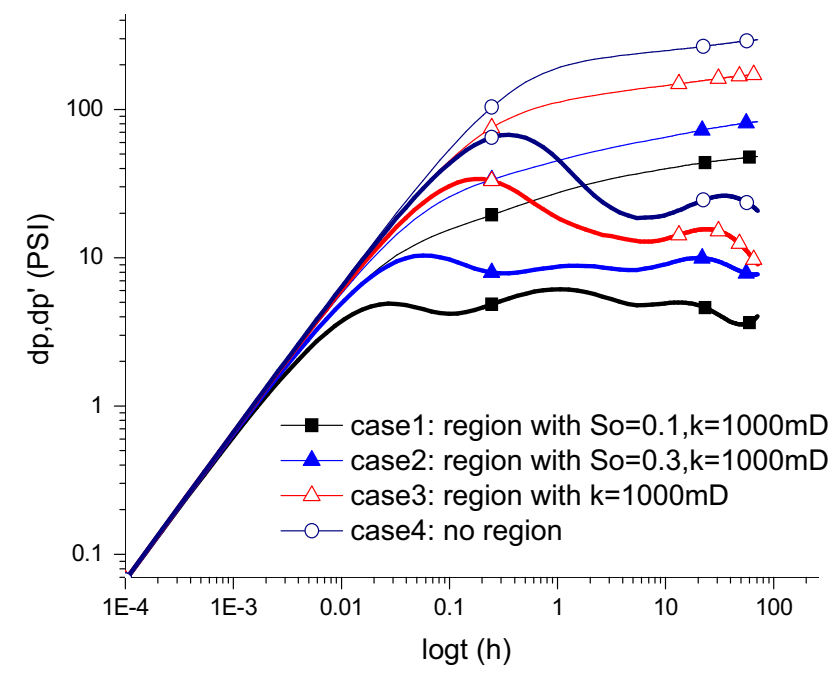

(a)

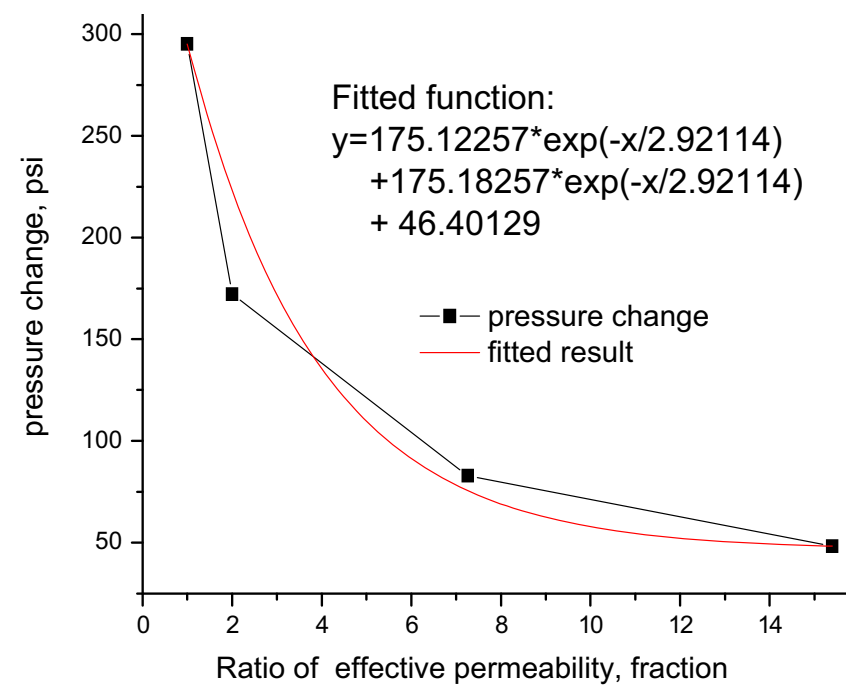

(b)

Fig. 5 Transient pressure response under constant pressure boundary condition. a Transient pressure response, b pressure change vs. ratio of $k \bullet k_{\mathrm{rw}}$ during buildup

Table 4 Wells data

\begin{tabular}{llllll}
\hline Time span (day) & Well $1\left(\mathrm{~m}^{3} /\right.$ day $)$ & Well $2\left(\mathrm{~m}^{3} /\right.$ day $)$ & Well $3\left(\mathrm{~m}^{3} /\right.$ day $)$ & Well $4\left(\mathrm{~m}^{3} /\right.$ day $)$ & Well $5\left(\mathrm{~m}^{3} /\right.$ day $)$ \\
\hline $0-60$ & 40 & -160 & 40 & 40 & 40 \\
$60-63$ & 0 & -160 & 60 & 50 & 50 \\
\hline
\end{tabular}

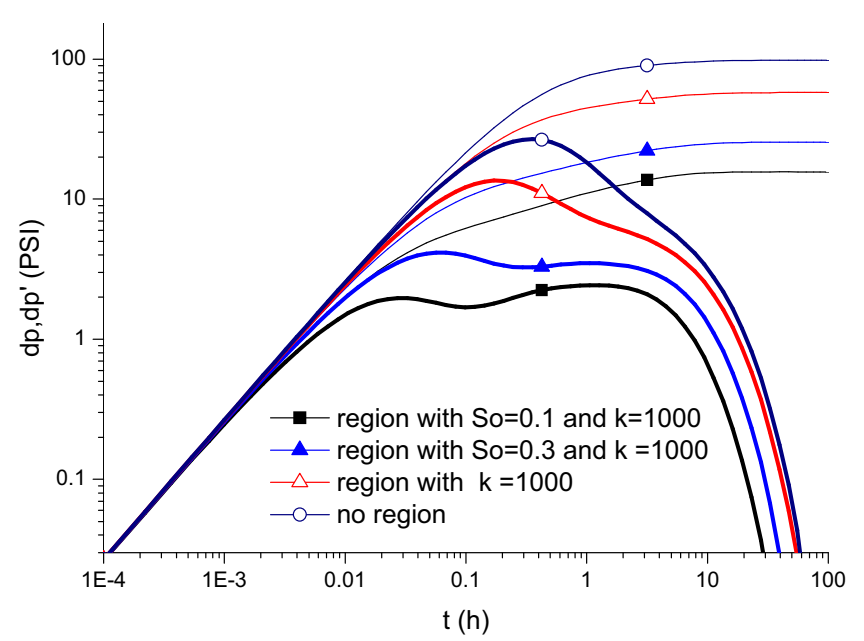

(a)

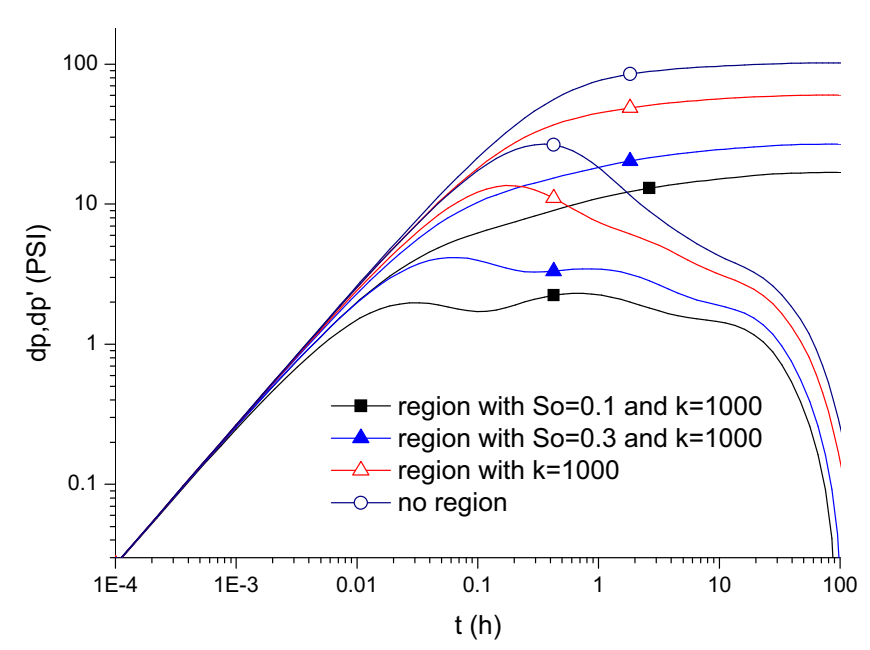

(b)

Fig. 6 Transient pressure response for the wells under constant rate. a Constant pressure boundary condition, b closed boundary condition

oil trapped in the low permeable zones. The viscosity difference between oil and water leads to viscous fingering, which makes water travel faster than oil. Polymer flood, injecting polymer solution with high viscosity into reservoir, is a very efficient to recover some bypassed oil in the mature reservoir. Daqing oil field has brought polymer flood into commercial application since 1996, achieving a largest scale successful application in the world, with 7,310 barrels of oil production per year attributed to polymer flood (Chen 2006; Dong et al. 2008).

In 2006, South block 5 was selected as alkaline-surfactant-polymer (ASP) pilot test. It has been developed more than 40 years and has entered the high water cut late development stage without having been flooded by 
polymer before. In order to evaluate the harm of chemical injection, some wells in South block 5 were selected to shut into measure the wellbore pressure. At first, shut-in pressure measurement was conducted during water flood, then followed polymer slug and lastly followed by ASP slug. The measured pressure in the water flood stage is used to evaluate to reservoir parameters and the late measured pressure is used to evaluate the influence of the chemical flood. This field case study is selected during water flood period.

\section{Thief zone identification}

In order to know the parameters of the reservoir better before polymer solution injection, well groups are selected to measure wellbore pressure during water flood period. The oil-water two-phase flow is applied. The relative permeability is given in Fig. 7 .

The well groups in South block 5 are selected as shown in Fig. 8, where there are 7 wells with average thickness of $5.8 \mathrm{~m}$.

The details about the wells are shown in Table 5. The first row is the well name. The second row is pressure change during shut-in. The fourth row is the time span of shut-in, which shows that buildup pressure of Well 1 is measured at March of 2006 year. The time of other wells is referred to the well 1. Although the seven wells are measured at different time, they also give us indications to judge the potential thief zone.

Table 5 shows that pressure change of production well 1 is about $160 \mathrm{psi}$, which is much smaller than the other production wells. However, its production is $120 \mathrm{~m}^{3} /$ day,

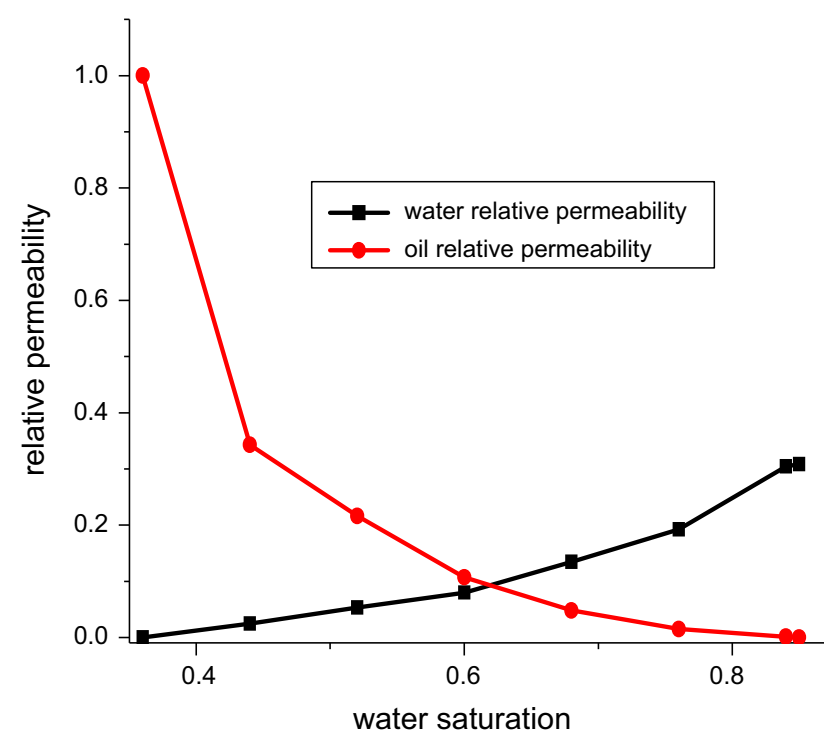

Fig. 7 Relative permeability curves which is much larger than other production wells. As to injection well 7 , its pressure change is only about 273 psi, which is much smaller than other injection wells, and at the same time its injection rate of $87 \mathrm{~m}^{3} /$ day also is larger than other injection wells.

Therefore, production well 1 has bigger production rate and smaller pressure change, while injection well 7 has bigger injection rate and smaller pressure change, which indicates that there is a high permeable zones connecting the two adjacent wells. Judging from fine geological study, there is a high permeability zone connecting the two wells and the absolute permeability difference is about two times. Incorporating with the transient pressure response, we judge that well 1 and well 7 are likely located in a thief zone, and the thief zone is mainly caused by water saturation difference.

Based on above understanding, we evaluate the thief zone. During the pressure history and pressure derivative matching, we add two regions including the well 1 and well 7 as shown in Fig. 8b. The two regions are used to simulate the gradual change of water phase saturation. Finally, we get the reservoir parameters as shown in Fig. 9.

The interpreted parameters are that: the inner region marked by red color has $S_{\mathrm{o}}=0.26, k=2300 \mathrm{mD}$ and $\phi$ $=0.3$, and the outer region marked by blue color is the transition area with $S_{\mathrm{o}}=0.42$. Apart from the properties in two regions, the ordinary reservoir has $S_{\mathrm{o}}=0.5$, $k=1000 \mathrm{mD}$ and $\phi=0.2$. The water saturation is evaluated based on water cut. In Daqing oil reservoir, high permeability always means large porosity. Therefore, the porosity of the inner region marked by red color is 0.3 .

The water effective permeability $k \cdot k_{\mathrm{rw}}$ in the red color region is about 8.93 times of water effective permeability in ordinary reservoir, among which about 3.9 times is caused by relative permeability difference decided by water saturation difference.

Figure 10 gives the matched results for the measured pressure data. We can see that according to the understanding of formation of the thief zone, the pressure is well fitted.

\section{Validation of the interpretations}

At about July of 2006, the injection well 7 began to inject polymer solution with polymer concentration of $1163 \mathrm{ppm}$. The measured polymer concentration of production well 1 at August and September is 0.0059 and $0.00661 \mathrm{~kg} / \mathrm{m}^{3}$, respectively, while measured polymer concentration of the well 3 is only $0.00004,0.00017 \mathrm{~kg} /$ $\mathrm{m}^{3}$. This means that there is higher effective mobility path linking production well 1 to injection well 7 , which shows that interpretation of a thief zone connecting well 1 and well 7 is correct. 


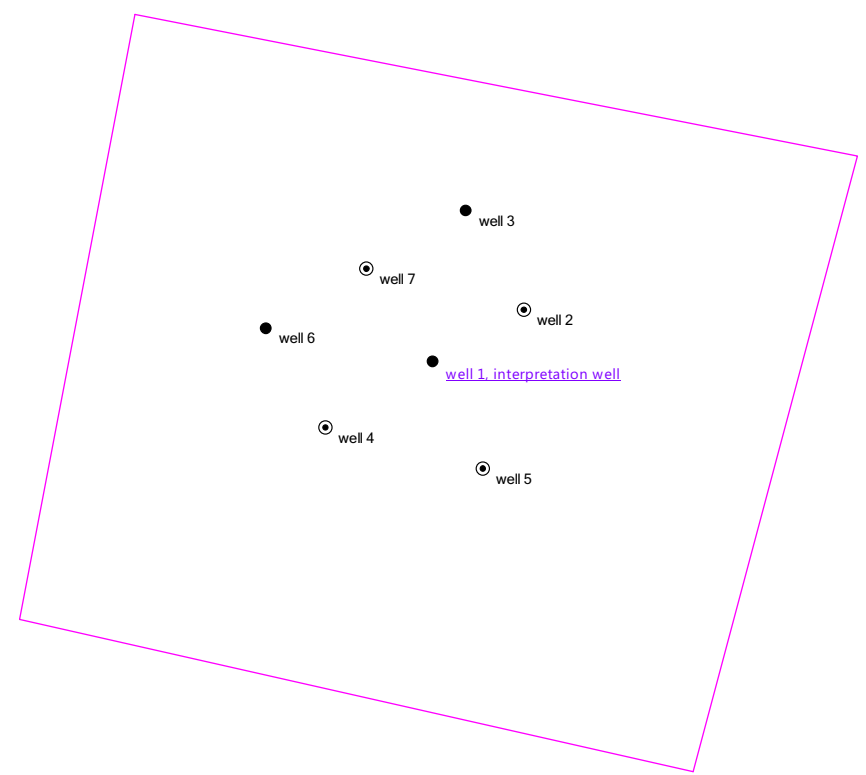

(a)

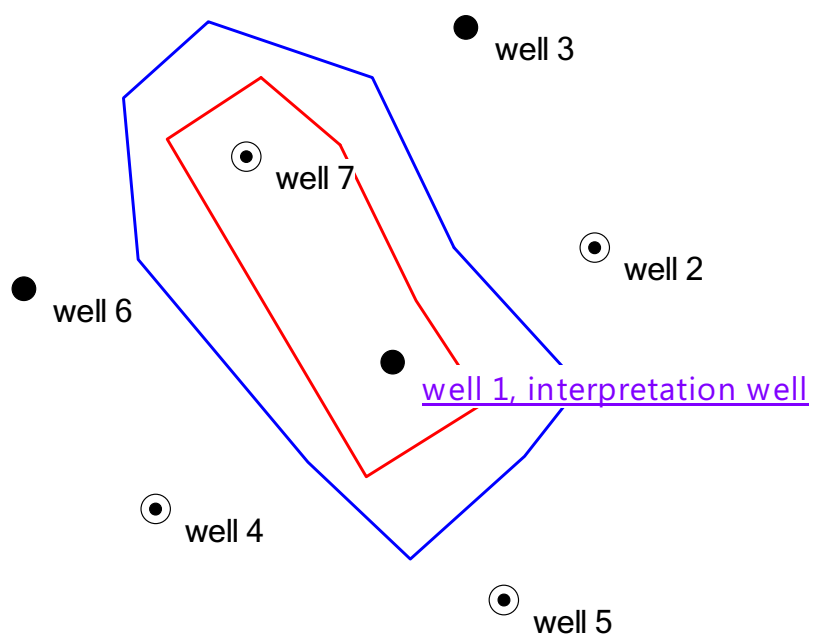

(b)

Fig. 8 Reservoir and wells. a Whole reservoir and wells, b partly enlarged

Table 5 Pressure change, rate, and measurement time of wells

\begin{tabular}{llllllll}
\hline Well name & Well 1 & Well 2 & Well 3 & Well 4 & Well 5 & Well 6 & Well 7 \\
\hline Pressure change, psi & 160 & -762 & 437 & -791 & -745 & 970 & -273 \\
Rate, $\mathrm{m}^{3}$ /day & 120 & -39.3 & 71 & -47 & -21 & 51 & -87 \\
Shut in time & March of 2006 & 86 days later & 78 days early & 75 days later & 51 days later & 3 days later & 50 days later \\
\hline
\end{tabular}

Negative sign means data of injection wells

\section{Discussion}

In this field case, formation of the interpreted thief zone is mainly determined by the water saturation difference. As mentioned before, the absolute permeability in the red color region is only 2.3 times of absolute permeability in ordinary reservoir, while the relative permeability $k_{\mathrm{rw}}$ in the red color region is 3.9 times of relative permeability $k_{\mathrm{rw}}$ in ordinary reservoir. Therefore, relative permeability $k_{\mathrm{rw}}$ decided by water saturation has more contribution to high mobility in the thief zone than the absolute permeability does, which indicates that uneven water saturation distribution induced by permeability heterogeneity is main contribution to formation of thief zone in mature reservoirs.

This also can explain the phenomena mentioned by Zhong et al. (2007). Zhong et al. report that some wells with the thief zone characteristics do not have water breakthrough at early stage; however, when injection pressure decrease at the late stage, injection rate of these wells is higher than before. Obviously, this cannot be simply explained by absolute permeability heterogeneity. This can be explained by variant of water saturation. Due to slow change of water saturation changes, water saturation in high permeable channels increase gradually. After long time, once the high mobility channel forms, the injection pressure decreases, and injection rate is also higher than before.

\section{Conclusions}

A new explanation of the formation for some kind of thief zone in mature reservoir is proposed, and numerical well test software based on PEBI gridding is used to study the characteristic of transient pressure response for the well located in the thief zone. Our conclusions could be summarized as below:

- The saturation difference is an important factor for the formation of some thief zone in mature reservoir. The absolute permeability heterogeneity is the initial factor. The areal permeability heterogeneity and water displacing lead to the uneven saturation distribution in mature reservoirs. The increase of the water saturation 


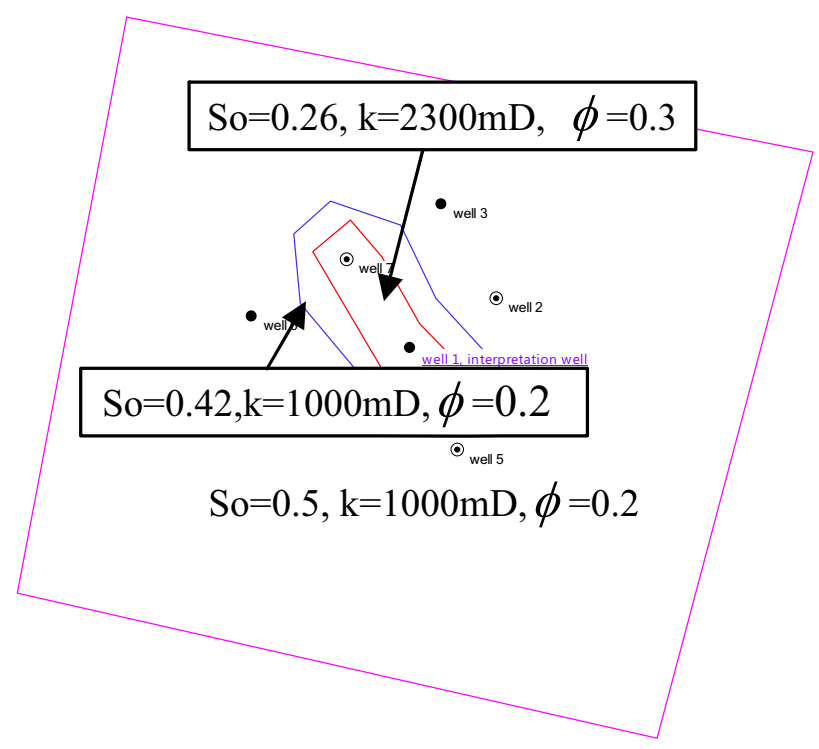

(a)

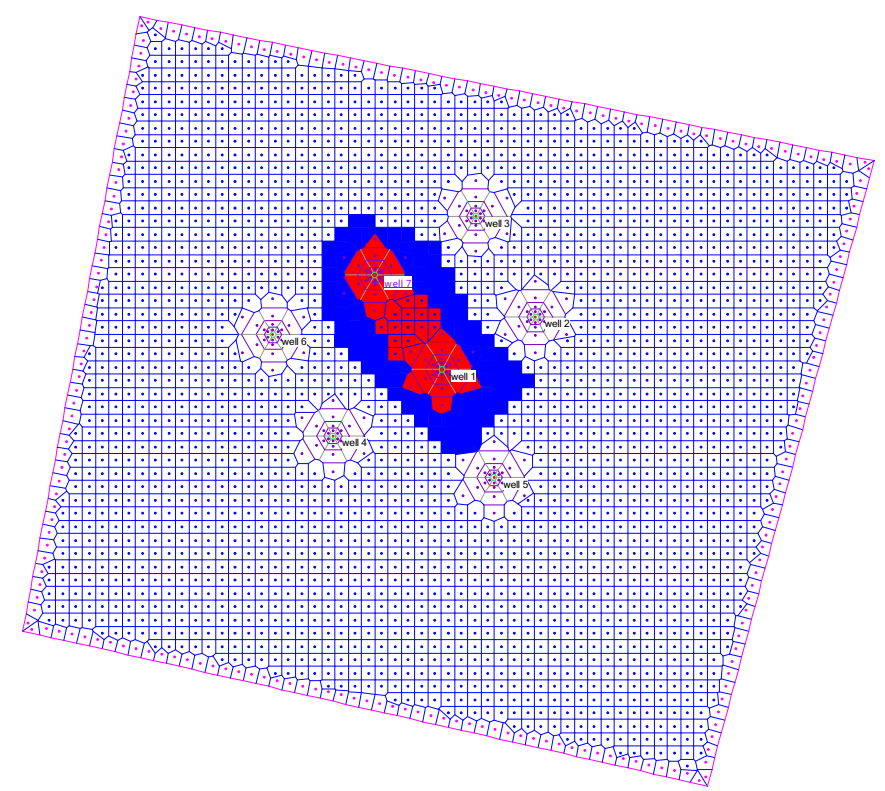

(b)

Fig. 9 Interpretation result, $S=0.4, k=1000 \mathrm{mD}, \phi=0.2$. a Reservoir and thief zone, $\mathbf{b}$ gridding

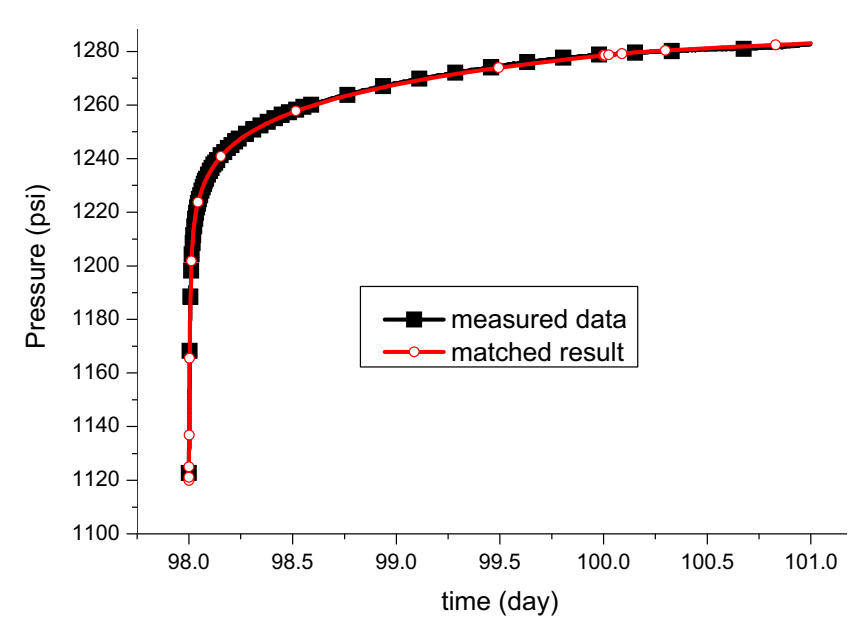

(a)

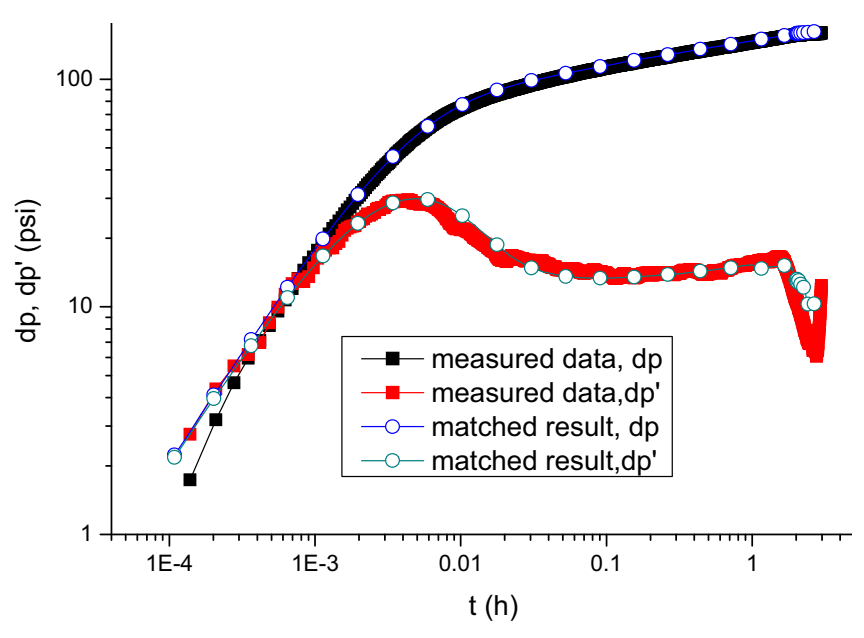

(b)

Fig. 10 Measured data and matched result. a Pressure history match, b pressure change and pressure derivative match

in the high permeable region leads to higher value of $k \cdot k_{\mathrm{rw}}$ in the high permeable region than the value of $k \cdot k_{\mathrm{rw}}$ in ordinary reservoir, and gradually the high mobility region becomes the thief zone.

- The well in the thief zone has small pressure change and its bump of the pressure derivative is not obvious.

- The results can help us to understand thief zones better and offer a new way to identify some kind of thief zone in mature reservoirs, which is not fractures or does not have a very big permeability difference compared to the surrounding formation.
Open Access This article is distributed under the terms of the Creative Commons Attribution 4.0 International License (http:// creativecommons.org/licenses/by/4.0/), which permits unrestricted use, distribution, and reproduction in any medium, provided you give appropriate credit to the original author(s) and the source, provide a link to the Creative Commons license, and indicate if changes were made.

\section{References}

Azim SA, Al-Ajmi H, Rice C, Bond D, Abdullah S, Laughlin B (2003) Reservoir description and static model build in 
heterogeneous Mauddud carbonates: Raudhatain Field, North Kuwait, SPE81524, paper presented at 13th Middle East Oil Show \& Conference held in Bahrain 5-8 April

Bane RK, Parker RA, Storbeck WG, Sunde RL (1994) Reservoir management of the Fullerton Clearfork Unit. SPE 27640, Permlan basin oil and gas recovery conference, Midland, Texas, USA, March

Chen JC (2006) EOR Technology improvement and its further development for Daqing oilfield during 10th 5-year-project. Petrol Geol Oilfield Dev Daqing 25(1):18-22

Chetri HB, Al-Anzi E, Al-Rabah A, Al-Dashti H, Al-Mutawa M, Chakravarthi R, Brown M, Isby J, Clark A (2003) Lessons learnt and experiences gained during two years of field monitoring, data integration and reservoir management: a case history of the Mauddud waterflood, North Kuwait. SPE 83961, Offshore Europe, Aberdeen, UK, September

Dong HZ, Fang SF, Wang DM et al (2008) Review of experience and management by polymer flooding at Daqing, SPE 114342 presented at the 2008 SPE/DOE improve oil symposium held in Tulsa, Oklahoma, USA, 19-23 April 2008

Felsenthal M, Gangle FJ (1975) A case study of thief zories in a California waterflood. J Petrol Technol 27:1385-1391

Feng QH, Wang S, Gao GQ, Li CY (2010) A new approach to thief zone identification based on interference test. J Petrol Sci Eng 75:13-18

Heinemann ZE, Brand CW, Munka M, Chen YM (1991) Modeling reservoir geometry with irregular grids. Soc Petrol Eng J 1991:225-232
Li DL, Xu CY, Wang JYL, Lu DT (2014) Effect of Knudsen diffusion and Langmuir adsorption on pressure transient response in shale gas reservoir. J Petrol Sci Eng 2014(124):146-154

Luo Y, Wang ZL, Nan GL (1999) A study on plugging technique in high permeable macro-pore path. Petrol Geology Oilfield Development in Daqing 18(5):39-41

Mahani H, Evazi M (2010) Vorticity-based perpendicular-bisector grids for improved upscaling of two-phase flow. SPE J 15(4):995-1008

Shawket G, Younes B, Moutaz S (2010) Thief zones and effectiveness of water-shut-off treatments under variable levels of gravity and reservoir heterogeneity in carbonate reservoirs. SPE EUROPEC/ EAGE annual conference and exhibition, Barcelona, Spain, June

Li BJ, Hamad N, Jim Lantz, Mansoor AR, Ihsan G, Mohammed AK (2008) Detecting thief zones in carbonate reservoirs by integrating borehole images with dynamic measurements, SPE 116286 annual technical conference and exhibition, 21-24 September 2008, Denver, Colorado, USA

Zha WS, Li DL, Lu DT, Kong XH (2008) PEBI grid division in interwell interference area. Acta Petrolei Sinica 29(5):742-746

Zhong DK, Zhu XM, Wu SH, Jin S, Jia DJ, Zhao Y (2007) Characteristics and control ing factors of high capacity channels of reservoirs at high water cut stage: a case from Block Hu 12 of Huzhuangji Oilfield. Petrol Explor Dev 34(2):207-211 